\title{
Performance Analysis of Image Segmentation Algorithm
}

\author{
Vishal Mehra $^{\# 1}$, S G Kerhalkar ${ }^{\# 2}$, N K Mittal ${ }^{\# 3}$ \\ ${ }^{\#}$ Oriental Institute of science and technology, Bhopal
}

\begin{abstract}
Image segmentation is a classic subject in the field of image processing and also is a hotspot and focus of image processing techniques. With the improvement of computer processing capabilities and the increased application of color image, the color image segmentation are more and more concerned by the researchers. In this paper we have proposed one new approach for Image segmentation which is based on Watershed algorithm followed by JND colour Histogram Algorithm. The Algorithm is implemented over MATLAB 7.9.0.
\end{abstract}

Keywords - Digital Image Processing, Image segmentation, Image Retrieval, watershed, Histogram

\section{INTRODUCTION}

Color image segmentation is useful in many applications. From the segmentation results, it is possible to identify regions of interest and objects in the scene, which is very beneficial to the subsequent image analysis or annotation.

The problem of segmentation is difficult because of image texture. If an image contains only homogeneous color regions, clustering methods in color space such as are sufficient to handle the problem. In reality, natural scenes are rich in color and texture. It is difficult to identify image regions containing colortexture patterns.

The approach taken in this work assumes the following:

- Each region in the image contains a uniformly distributed color-texture pattern.

- The color information in each image region can be represented by a few quantized colors, which is true for most color images of natural scenes.

- The colors between two neighboring regions are dis- tinguishable - a basic assumption of any color image segmentation algorithm.

Image retrieval is a technique used for retrieving similar images from an image database. In the past few years, research in colour-based image and video retrieval has presented acceptable results and commercial systems have already been published in various application domains which require retrieval of images from an image collection. Very often, applications require retrieval of images where the query object or region cover only a fractional part of the database image, a task essentially identical to appearance-based object recognition with unconstrained background. Retrieval and recognition based on object appearance must take into account the factors that influence the formation of colour images. Recorded colours depend on the viewing geometry, the illumination conditions, the sensor spectral sensitivities and the object's surface reflectances. In particular, illumination colour, intensity as well as view point and background may change in many applications. Other not so well modelled or not readily measured effects like mutual illumination and individual camera characteristics (e.g. gamma correction settings) also result in variations of object appearance. Moreover, partial occlusion and deformation of non-rigid objects must also be taken into consideration. Consequently, invariance or at least robustness to the above diverse factors is highly desirable.

Large image databases are difficult to browse with text search, if the user wants to search images which have a similar color content. Retrieval based on color matching solves this problem.

\section{BACKGROUND WORK AND RELATED METHODS}

Various approaches have been developed for image segmentation, and image retrieval. Few approaches referred in our literature are discussed below:

Ozden.M, Polat.E in 2006 describes a new color image segmentation method based on low-level features including color, texture and spatial information [1]. The mean-shift algorithm with color and spatial information in color image segmentation is in general successful, however, in some cases, the color and spatial information are not sufficient for superior segmentation. They used wavelet frames that provide translation 
invariant texture analysis. Their proposed method integrates additional texture feature to the color and spatial space of standard mean-shift segmentation algorithm.

Tang. $\mathrm{J}$ in 2010 proposed that proposes a color image segmentation method of automatic seed region growing on basis of the region with the combination of the watershed algorithm with seed region growing algorithm which based on the traditional seed region growing algorithm [2].

\section{A. The Basic Principle of Seed Region Growing Algorithm}

The basic idea of region growing method is a collection of pixels with similar properties to form a region. The steps are as follows: First, we need to find a seed pixel as a started point for each of needed segmentation. And then merge the same or similar property of pixel (Based on a pre-determined growing or similar formula to determine) with the seed pixel around the seed pixel domain into the domain of seed pixel. These new pixels as a new seed pixel to continue the above process until no more pixels that satisfy the condition can be included.

In the practical application of this method we need to address three questions:

a) Chose or determined a group of seed pixel which can correctly represent the required region;

b) Fixed the formula which can contain the adjacent pixels in the growth;

c) Made rules or conditions to stop the growth process.

The seed region growing algorithm is proposed by Adams and Bischof, Metmert and Jackway further described the dependency relationship between pixels in the seed growth:

a) The first order of dependence occurs when the number of pixels has the same difference ratio as their vicinity.

b) The second order of dependence occurs when a pixels has the same difference ratio as their vicinity.

\section{B. Watershed Image Segmentation Methods}

The watershed algorithm is more representative in the application of mathematical morphology theory for image segmentation. Watershed algorithm is a region-based segmentation techniques image that uses image morphology. Watershed algorithm is an iterative adaptive threshold algorithm. The idea of watershed algorithm is from geography (shown in Figure 2), it see gradient magnitude image as a topographic map, the gradient magnitude in correspond with altitude, the different gradient in correspond with the peak and basin in valley in the image. It sees every object of image (including background) as a separate part and requested there must have one tag at least in the each object (or seed points). Marker is knowledge about the object based on application-oriented; it is selected by the operator manually or by automatic process. The objects can use watershed algorithm to transform and develop regional growth after maker.

Belongie. S, and his colleague in 1998 describe a system that uses the blobworld representation to retrieve images [3]. An important and unique aspect of the system is that, in the context of similarity-based querying, the user is allowed to view the internal representation of the submitted image and the query results. Similar systems do not offer the user this view into the workings of the system; consequently, the outcome of many queries on these systems can be quite inexplicable, despite the availability of knobs for adjusting the similarity metric.

Geethalakshmi. S. N, and Jothi.T in 2010 works on Medical imaging and analysis. Image segmentation and enhancement is one of the central research themes in medical image analysis and with the increasing number of imaging analysis and imaging studies, the necessity for automated medical image segmentation methods are expanding. Their research work focus on segmentation that combines neural network with Watershed algorithm modified to use automatic optimal threshold selection algorithm. The problem of over-segmentation is solved by using preprocessing technique. Experimental results prove that the proposed method is efficient in segmenting medical images [4].

Navon. E in 2004 proposed a method of image segmentation which was based on adaptive local thresholds [5]. Their proposed algorithm divides the image into homogeneous regions by local thresholds. The number of thresholds and their values are adaptively derived by an automatic process, where local information is taken into consideration. First, the watershed algorithm is applied. Its results are used as an initialization for the next step, which is iterative merging process. During the iterative process, regions are merged and local thresholds are derived. The thresholds are determined one-by-one at different times during the merging process. Every threshold is calculated by local information on any region and its surroundings. 
Kishor Bhoyar \& Omprakash Kakde in 2010 proposed a new algorithm for Image segmentation which was based on JND colour histogram. In their proposed model Histogram of the given color image is computed using JND color model [6]. This samples each of the three axes of color space so that just enough number of visually different color bins (each bin containing visually similar colors) are obtained without compromising the visual image content. The number of histogram bins are further reduced using agglomeration successively. This merges similar histogram bins together based on a specific threshold in terms of JND. This agglomerated histogram yields the final segmentation based on similar colors.

In the past decade, many image retrieval systems have been developed, such as the IBM QBIC system developed at the IBM Almaden Research Center, the VIRAGE System developed by the Virage Incorporation, the Photobook System developed by the MIT Media Lab, the VisualSeek system developed at Columbia University, the WBIIS System developed at Stanford University, and the Blobworld System developed at U.C. Berkeley. Existing color-based general-purpose image retrieval systems roughly fall into three categories depending on the signature extraction approach used: histogram, color layout, and region-based search.

\section{Few approaches are discussed below:}

P. S. Hiremath and Jagadeesh Pujari in 2010 proposed a method for Image Retrieval based on Color, Texture and Shape features using Image and its complement presents a novel framework for combining all the three i.e. color, texture and shape information, and achieve higher retrieval efficiency using image and its complement [7]. The image and its complement are partitioned into non-overlapping tiles of equal size. The features drawn from conditional co-occurrence histograms between the image tiles and corresponding complement tiles, in RGB color space, serve as local descriptors of color and texture. This local information is captured for two resolutions and two grid layouts that provide different details of the same image. The combination of the color and texture features between image and its complement in conjunction with the shape features provide a robust feature set for image retrieval.

Konstantinidis. K et.al in 2005 proposed a Image retrieval method based on fuzzy color histogram processing. A new histogram creation method has been proposed [8]. The histogram is created based on the $\mathrm{L}^{*} \mathrm{a} \mathrm{b}^{*}$ color space components which are considered to be fuzzy sets. The proposed histogram is acquired through the linking of these fuzzy sets according to 27 fuzzy rules. Histogram-based retrieval system requires the following components in order to work: A suitable color space such as HSV, L*a*b* or L* $\mathrm{u}^{*} \mathrm{v}^{*}$, a histogram representation such as classic [9, 10] or joint [11] histograms, and a similarity metric like the L2 (Euclidean Distance), the Matusita distance [12] or the Histogram Intersection method [13].

Jeong. S in 2001 present a report on image retrieval, and proposed a method of image retrieval based on Histogram Based [14]. In his project, six histogram-based search methods are investigated in two different color spaces, RGB and HSV. Histogram search characterizes an image by its color distribution, or histogram but the drawback of a global histogram representation is that information about object location, shape, and tex ture is discarded. Thus this project showed that images retrieved by using the global color histogram may not be semantically related even though they share similar color distribution in some results. An image retrieval demo system was built to make it easy to test the retrieval performance and to expedite further algorithm investigation. And six histogram-based image retrieval methods in two color spaces were exhaustively compared by providing precision vs. recall graphs for each image class and for all test images. In general, histogram-based retrievals in HSV color space showed better performance than in RGB color space.

\section{ALGORITHM FOR IMAGE SEGMENTATION}

In this paper we will focus on Image segmentation based on Watershed and colour Histogram and Image retrieval based on Tonal distribution.

Watershed segmentation is a morphological based method of image segmentation. The gradient magnitude of an image is considered as a topographic surface for the watershed transformation. Watershed lines can be found by different ways. The complete division of the image through watershed transformation relies mostly on a good estimation of image gradients. The result of the watershed transform is degraded by the background noise and produces the over-segmentation. Also, under segmentation is produced by low-contrast edges generate small magnitude gradients, causing distinct regions to be erroneously merged.

Watershed segmentation is a predominant segmentation scheme with several advantages. It ensures the closed region boundaries and gives solid results. It is a way of automatically separating or cutting apart particles that touch. The watershed algorithm uses concepts from mathematical morphology [15] to partition images into homogeneous regions [16]. The general process of the conventional watershed algorithm consists of five steps as given : 


\section{Step 1 : Load the Image \\ Step 2 : Preprocess the image \\ Step 3 : Marker Extraction \\ Step 4 : Segmentation process \\ Step 5 : Threshold adjustment and final result}

\section{JND COLOUR HISTOGRAM}

The JND color model in RGB space based on limitations of human vision perception as proposed. The human retina contains two types of light sensors namely; rods and cones, responsible for monochrome i.e. gray vision and color vision respectively. The three types of cones viz, Red, Green and Blue respond to specific ranges of wavelengths corresponding to the three basic colors Red, Green and Blue. The concentration of these color receptors is maximum at the center of the retina and it goes on reducing along radius. According to the three color theory of Thomas Young, all other colors are perceived as linear combinations of these basic colors. According to [12] a normal human eye can perceive at the most 17,000 colors at maximum intensity without saturating the human eye. In other words, if the huge color space is sampled in only 17,000 colors, a performance matching close to human vision at normal illumination may be obtained. A human eye can discriminate between two colors if they are at least one 'just noticeable difference (JND)' away from each other. The term 'JND' has been qualitatively used as a color difference unit.

\section{Computing JND histogram}

i) Initialize two data structures Table 1 and Table 2. Initialize the first entry in Table 1 by the first color vector in the image i.e. top left pixel color vector $[R, G, B]$ and the frequency(population) by one. Initialize the first entry in Table 2 by the current row index value of Table 1 i.e. 1 , and the top left pixel position row and column i.e. $y($ column $)=1$ and $x($ row $)=1$. Also initialize a (row, column) pointer to top left corner of the image. Select a proper similarity threshold $\Theta$ depending on the precision of vision from fine to broad as required by the application.

ii) Read the next pixel color vector in scan line order.

iii) Compare the new pixel color vector with all the previous entries in Table 1 one by one and if found similar to any of them, then accommodate it in the respective bin. Update Table 2 by entering the current index and the current row and column values and go to step $v$.

$i v$ If the new color vector is not equal to any of the previously recorded color vectors in Table 1, increment the row index of Table 1, enter the new color vector in it, set the population to 1, make the index, row and column entry in Table 2 and go to step ii.

v) Repeat step ii) to iv) for all the pixels in the image.

vi) Sort Table 2 in the increasing order of the color index.

vii) Save the Table 1 and Table 2 for latter analysis of the histogram.

\begin{tabular}{|l|l|l|l|l|}
\hline $\begin{array}{l}\text { JND Color } \\
\text { Index }\end{array}$ & $\mathrm{R}$ & $\mathbf{G}$ & $\mathbf{B}$ & $\mathbf{H}$ \\
\hline 1 & 5 & 15 & 20 & 335 \\
\hline 2 & 10 & 100 & 20 & 450 \\
\hline 3 & 20 & 50 & 10 & 470 \\
\hline$\cdot$ & $\cdot$ & $\cdot$ & $\cdot$ & $\cdot$ \\
\hline $\mathrm{K}$ & 25 & 72 & 90 & 200 \\
\hline & & & & \\
\hline
\end{tabular}

TABLE 1: Color population

\begin{tabular}{|l|l|l|}
\hline $\mathbf{X}$ & $\mathbf{Y}$ & $\begin{array}{l}\text { JND Color Index for } \\
\left(\mathrm{x}_{\mathrm{i}}, \mathrm{y}_{\mathrm{i}}\right) \text { from Table 1 }\end{array}$ \\
\hline $\mathrm{X}_{1}$ & $\mathrm{y}_{1}$ & 1 \\
\hline $\mathrm{x}_{1}$ & $\mathrm{y}_{2}$ & 1 \\
\hline- & $\cdot$ & $\cdot$ \\
\hline $\mathrm{x}_{\mathrm{i}}$ & $\mathrm{y}_{\mathrm{i}}$ & $\mathrm{K}$ \\
\hline- & $\cdot$ & $\cdot$ \\
\hline
\end{tabular}

TABLE 2: Color index-pixel location relation

\section{Colour Based Image Retrieval:}

Large image databases are difficult to browse with text search, if the user wants to search images which have a similar color content. Retrieval based on color matching solves this problem. Color based matching evaluation can be made using the following steps:

1. If a query image has already been analyzed and data stored into a database, jump to 7.

2. Read the query image from the disk.

3. Make a color space conversion if needed. 
4. Analyze the query image pixel by pixel and store the values in a quantized histogram.

5. Change bin hit values to relative value (percentage).

6. Save the histogram data with additional information average color, dominant colors) and image name in the database.

7. Calculate color differences and histogram difference on a higher level ( 8 - 64 bins) between query image and database images.

8. Select the best candidates to closer evaluation (64 - 512 bins).

9. Select matching images.

\section{PROPOSED WORK AND ALGORITHM}

The proposed work can be divided into the main two steps:

A. Image segmentation Part

1. Insert the Image

2. Pre-processing of input Image

3. Apply watershed algorithm on the input image

4. Calculate colour Histogram of the output of step no.3

5. Save the record in database

\section{B. Image Retrieval Part}

1. Query the image.

2. Calculate the Histogram of the Input Image.

3. Calculate the similarity measure by matching the histogram of input image with the template result saves in the database.

4. Image matched with the queried image get back to the user as a resultant image.

The flow chart of the proposed work is shown below:
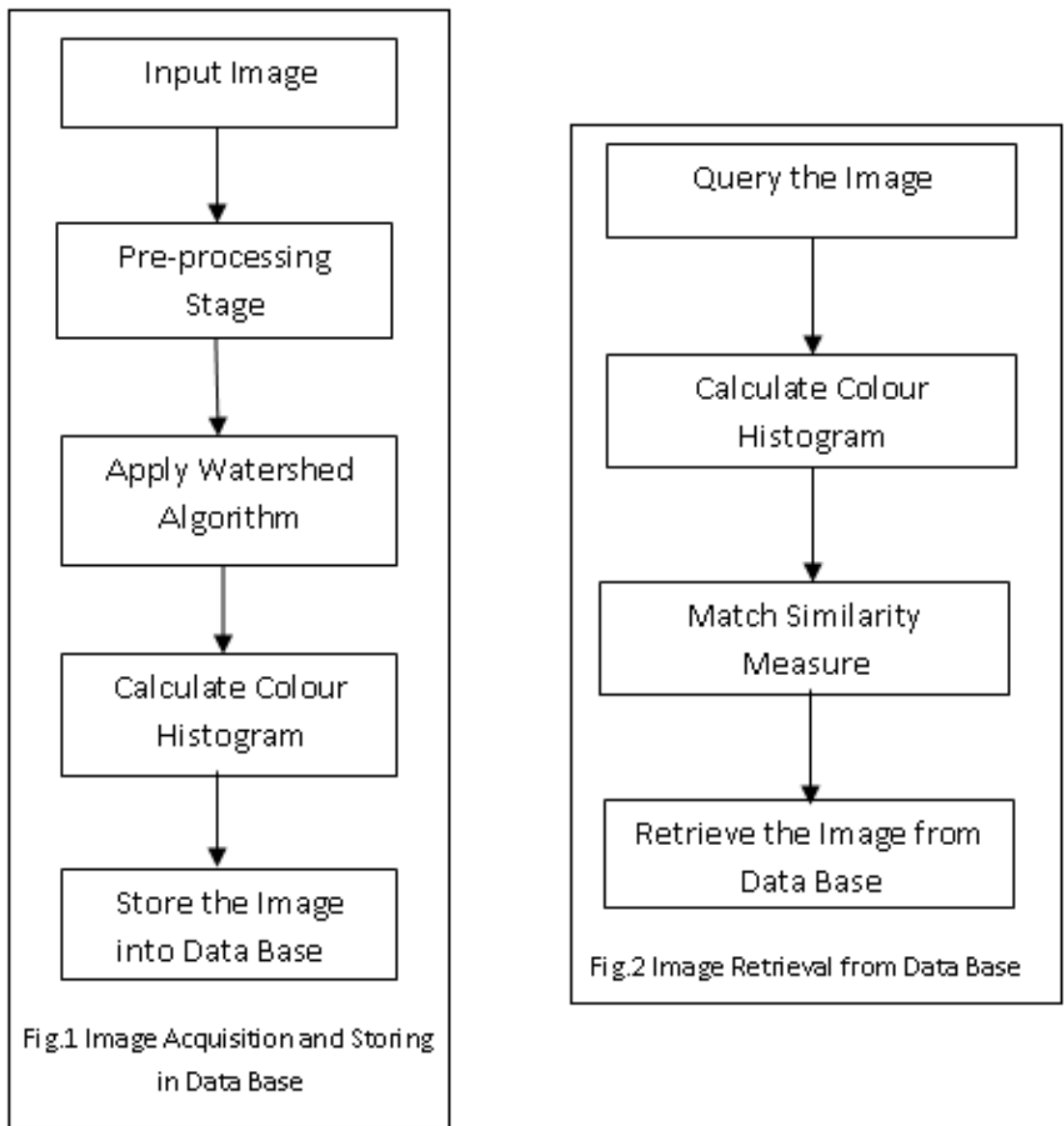


\section{CONCLUSION AND FUTURE WORK}

In this paper we review various approaches to colour image segmentation, two algorithms out of these are described detailed named, watershed algorithm and Colour Histogram approach. The algorithm is robust for large variety of colour images. The future work may includes the hybrid image segmentation algorithm, which can be a combination of two or more algorithm, as shown in proposed algorithm. Proposed algorithm will be test for various kinds of image database, which will further show the efficiency and robustness of the proposed algorithm.

\section{REFERENCES}

[1] Ozden M, Polat E, “A color image segmentation approach for content based image retrieval”, The Journal of the Pattern Recognition society, pp. 1318-1325, 2007.

[2] Tang. J, "A Color Image Segmentation algorithm Based on Region Growing", 2nd International Conference on Computer Engineering and Technology, pp. 634-637, IEEE 2010.

[3] Belongie. S, Carson. C, Greenspan.H, Malik.J, "Color- and Texture-Based Image Segmentation Using EM and Its Application to Content-Based Image Retrieval”, ICCV '98, pp.1-8, 1998.

[4] Geethalakshmi. S. N, Jothi. T, "Segmentation based on Enhanced morphological watershed Algorithm", Journal of Global Research in Computer Science, pp.75-82, 2010.

[5] Navon. E, Miller. O, Averbuch. A, "Color image segmentation based on adaptive local thresholds", pp.1-17, Image and Vision Computing 23 (2005), Science Direct.

[6] Bhoyar. K, Kakde. O, "Color Image Segmentation based on JND color Histogram", International Journal of Image Processing (IJIP) Volume(3), Issue(6), pp.283-292, 2010.

[7] Hiremath. P. S, Pujari. J, "Content Based Image Retrieval based on Color, Texture and Shape features using Image and its complement", International Journal of Computer Science and Security, Volume (1) : Issue (4), pp.25-35, 2010.

[8] Konstantinidis. K, Gasteratos. A, Andreadis. I, "Image retrieval based on fuzzy color histogram processing", Optics Communications 248 (2005) 375-386, Science Direct.

[9] K. Konstantinidis, I. Andreadis, J. Comput. Methods Sci. Eng. (JCMSE), to appear.

[10] Y. Liang, H. Zhai, P. Chavel, Opt. Commun. 212 (2002) 247.

[11] G. Pass, R. Zabih, Multimed. Syst. 7 (3) (1999) 234.

[12] K. Fukunaga, Introduction to Statistical Pattern Recognition, second ed., Academic Press, New York, 1990.

[13] M.J. Swain, D.H. Ballard, Int. J. Comput. Vis. 7 (1991).

[14] Jeong. S, "Histogram-Based Color Image Retrieval”, Psych221/EE362 Project Report, pp.1-21, 2001.

[15] Gonzalez, R.C. and Woods, R.E. (2001) Digital Image Processing, Prentice Hall, New Jersey, USA, Pp. 567-635.

[16] Vincent, L. and Soille, P. (1991) Watersheds in digital spaces: An efficient algorithm based on immersion simulations, IEEE Trans. Pattern and Machine Intelligence, Vol. 13, Pp. 583-598.

[17]A. C. Guyton, “A text book of medical Physiology”, W.B.Saunders company, Philadelphia, pp.784-824, (1976). 